\title{
Space-time variation of the fine-structure constant and evolution of isotope abundances
}

\author{
M. G. Kozlov* \\ Petersburg Nuclear Physics Institute, Gatchina, 188300, Russia \\ V. A. Korol \\ St. Petersburg Polytechnical University, St. Petersburg, Russia \\ J. C. Berengut, V. A. Dzuba, and V. V. Flambaum \\ University of New South Wales, Sydney, Australia
}

(Received 28 July 2004; revised manuscript received 13 September 2004; published 14 December 2004)

\begin{abstract}
At present several groups are analyzing astrophysical data in a search for the time variation of the finestructure constant $\alpha$. Here we discuss how to exclude systematic effects caused by changes in the isotope abundances during the evolution of the universe. We suggest using particular combinations of the transition frequencies of $\mathrm{O}_{\text {II, }} \mathrm{Al}_{\text {II, }} \mathrm{Al}$ III, $\mathrm{Si}_{\text {III }}$ and $\mathrm{Mn}_{\text {II }}$ as anchors, which are insensitive to $\alpha$ variation and to changes in isotope abundances. These anchors can be used to determine the cosmological redshift. Then, one can use other combinations of frequencies as probes for the time variation of $\alpha$ and another set as probes for the isotopic abundances. In this way it is possible to eliminate one source of systematic errors in the search for $\alpha$ variation and get more information about evolution of the isotopes. On the level of accuracy that has already been reached in the search for $\alpha$ variation it is possible to see $\sim 10 \%$ changes in isotope abundances of $\mathrm{Si}$ and Ni.

DOI: 10.1103/PhysRevA.70.062108

PACS number(s): 06.20.Jr, 31.30.Gs, 98.80.Ft
\end{abstract}

\section{INTRODUCTION}

If, during previous stages of the evolution of the universe, the fine-structure constant $\alpha$ was different from its present value $\alpha_{0}$, we can expect small deviations of atomic frequencies from their laboratory values [1]:

$$
\begin{gathered}
\omega=\omega_{\mathrm{lab}}+q x+O\left(x^{2}\right) \\
\approx \omega_{\mathrm{lab}}+2 q \Delta \alpha / \alpha, \\
x \equiv\left(\alpha / \alpha_{0}\right)^{2}-1,
\end{gathered}
$$

where the parameter $q$ rapidly grows with the nuclear charge $Z$. At least three groups are looking for such deviations in the spectra of distant quasars [2-6]. These groups use different data and their results are not fully consistent with each other. In [2-4] a nonzero $\alpha$ variation is reported, while [5,6] agree with the constant $\alpha$

$$
\frac{\Delta \alpha}{\alpha}=10^{-5} \times\left\{\begin{array}{cc}
-0.57 \pm 0.11 & {[4],} \\
-0.04 \pm 0.19 \pm 0.27_{\text {sys }} & {[5],} \\
-0.06 \pm 0.06 & {[6]}
\end{array}\right.
$$

The frequency shifts which correspond to Eq. (2) are of the order of magnitude of the typical isotope shifts. Therefore, any changes in isotope abundances can cause systematic errors in the search for $\alpha$ variation (see, for example, discussions in [4,7-9] and references therein).

Here we use recent calculations of the isotope shifts [10-12] to reanalyze the role of the isotope evolution in the

\footnotetext{
*Electronic address: mgk@MF1309.spb.edu
}

search for $\alpha$ variation. We suggest how to exclude the possible systematic error caused by isotope shift from the search for $\alpha$ variation. We also show that the spectroscopic data from Eq. (2) can be used to get information about evolution of isotope abundances of $\mathrm{Si}$ and $\mathrm{Ni}$.

To a good approximation the shift in frequency of any transition in an isotope with mass number $A^{\prime}$ with respect to an isotope with mass number $A$ can be expressed as

$$
\Delta \omega^{A^{\prime}, A}=k_{\mathrm{MS}}\left(\frac{1}{A}-\frac{1}{A^{\prime}}\right)+F \delta\left\langle r^{2}\right\rangle^{A^{\prime}, A},
$$

where $\left\langle r^{2}\right\rangle$ is the mean square nuclear radius. The mass shift constant $k_{\mathrm{MS}}$ accounts for the normal mass shift and for the specific mass shift [13], $k_{\mathrm{MS}}=k_{\mathrm{NMS}}+k_{\mathrm{SMS}}$, where

$$
k_{\mathrm{NMS}} \approx \frac{\omega}{1823},
$$

and the value 1823 refers to the ratio of the atomic mass unit (amu) to the electron mass. The values of the constants $k_{\text {SMS }}$ for the specific mass shift and $F$ for the volume shift in Eq. (3) depend on the details of the atomic structure (see $[10,11]$ for details). Note that in this paper we use the common sign convention with $k_{\mathrm{NMS}}>0[13,14]$, which differs from the convention used in $[10,11]$ and some other publications.

The present accuracy of the theory for $k_{\mathrm{SMS}}$ for atoms with more than one valence electron is not always sufficient for our purpose. However, it is possible to improve the theory by applying techniques suggested in $[15,16]$. Note that calculations for the ions with one electron above closed shells are already sufficiently accurate $[10,17,18]$. Below we ignore the volume shift of Eq. (3). For light elements it is much smaller than the mass shift, but becomes more impor- 
tant for such elements as $\mathrm{Ni}, \mathrm{Zn}$, and Ge. Still, its size is comparable to the accuracy to which we currently know the isotope shift parameters $k_{\mathrm{MS}}$.

\section{ISOTOPIC EFFECTS}

In this section we discuss the implications of recent calculations of isotope shifts [10-12] for the astrophysical search for $\alpha$ variation. Table I presents calculated values of the parameters $q$ and $k_{\mathrm{MS}}$ for the transitions used in astrophysical surveys [2-6]. In order to estimate possible isotope shifts in astrophysics we calculate isotope shifts $\Delta \omega_{1,2}$ for two simple assumptions.

(1) $\Delta \omega_{1}$ corresponds to the case when the abundance of the leading isotope $A$ is reduced by $10 \%$, while the abundance of the next to leading isotope $A^{\prime}$ is increased by the same amount. That means that for $\mathrm{Si}$ and $\mathrm{Cr} A^{\prime}=A+1$; for $\mathrm{Mg}, \mathrm{Ni}$, and $\mathrm{Zn} A^{\prime}=A+2$; for $\mathrm{Ti}, \mathrm{Fe}$, and $\mathrm{Ge} A^{\prime}=A-2$. Then $\Delta \omega_{1}=0.1 \times \Delta \omega^{A^{\prime}, A}$.

(2) $\Delta \omega_{2}$ corresponds to the substitution of the terrestrial abundances with the single leading isotope. For $\mathrm{O}, \mathrm{Al}$, and Mn that means zero shifts as these elements have no isotopes.

The shifts $\Delta \omega_{1}$ and $\Delta \omega_{2}$ are usually of the opposite sign, and we use them to estimate the range of the possible isotopic shifts for distant absorber. In order to compare isotopic effects with the expected effects from $\alpha$ variation, Table I also presents the shifts $\Delta \omega_{\alpha}$ which correspond to $\Delta \alpha / \alpha=$ $-0.57 \times 10^{-5}$ [4]. Note that our first assumption of a $10 \%$ change in abundances is a rather conservative one.

Sometimes, much more dramatic scenarios of isotope evolution are considered (see, for example, [9] and the references therein). It is easy to use data from Table I to calculate isotope shifts for any assumptions about isotope evolution. For example, consider a third scenario with extremely large isotopic effects, when all abundance is transferred to the least abundant isotope. In this case isotopic effects are typically an order of magnitude larger compared to Table I:

$$
\Delta \omega_{3} \approx \Delta \omega_{1} \times\left\{\begin{array}{cc}
4 & \text { for } \mathrm{Mg}, \\
20 & \text { for } \mathrm{Si}, \mathrm{Cr}, \mathrm{Ni}, \text { and } \mathrm{Zn}, \\
-10 & \text { for } \mathrm{Ti} \text { and } \mathrm{Fe} .
\end{array}\right.
$$

For such dramatic scenarios our conclusions will only be enhanced.

Table I shows that the shifts $\Delta \omega_{1,2}$ are indeed of the same order of magnitude as the shifts observed in [4]. Moreover, in some cases there is a strong correlation between $\Delta \omega_{\alpha}$ and $\Delta \omega_{1,2}$. That confirms earlier suggestions that isotopic effects may lead to significant systematic errors in the search for $\alpha$ variation.

Let us, for example, consider Fe II. Here isotope shifts are relatively small. On the other hand, the coefficients $q$ and $k_{\mathrm{MS}}$ are strongly correlated, and it is impossible to disentangle these two effects. For the lines used in [5], the isotope shifts which correspond to our two limiting cases would imitate the following $\alpha$ variation:

$$
\frac{\Delta \alpha}{\alpha}=10^{-5} \times \begin{cases}-0.14, & \text { case } 1, \\ +0.07, & \text { case } 2 .\end{cases}
$$

These values lie within the error bars given by Quast et al., but will not allow one to improve their results significantly, unless isotope effects are accounted for. That, in turn, will require one to include other lines in the analysis.

\section{ANCHORS AND PROBES}

When spectra of distant astrophysical objects are observed, one needs to exclude the cosmological redshift. That can be done by looking at the frequency ratios for different transitions, i.e., by comparing $\left(\omega_{i} / \omega_{k}\right)_{\text {astro }}$ with $\left(\omega_{i} / \omega_{k}\right)_{\text {lab }}$. When forming these ratios it is convenient to use "anchor" lines, which are not sensitive to the variation of the parameter of interest.

As we saw above, there can be two effects of the same order of magnitude, i.e., possible $\alpha$ variation and variation of isotope abundances. Therefore, we need anchors which are insensitive to both effects. In Table I there is only one line of $\mathrm{Si}$ II, which may be considered suitable as anchor:

$$
A_{I}=\omega\left({ }^{2} P_{1 / 2}^{o} \rightarrow{ }^{2} S_{1 / 2}\right)_{\mathrm{Si} \mathrm{II}}=65495 \mathrm{~cm}^{-1} .
$$

It is preferable to have more than one anchor. However, all other lines have either relatively large $q$, or $k_{\mathrm{MS}}$, or both. Aluminum, oxygen, and manganese ions have only one stable isotope each (the leading isotope abundance for ${ }^{16} \mathrm{O}$ is greater than 99\%). In order to exclude the $\alpha$ dependence of transition frequencies we can use the following combinations of ${ }^{16} \mathrm{O}$ and ${ }^{27} \mathrm{Al}$ :

$$
\begin{aligned}
A_{I I} & =0.62 \omega\left({ }^{4} S_{3 / 2}^{o} \rightarrow{ }^{4} P_{5 / 2}\right)_{\mathrm{O} \mathrm{II}}-0.38 \omega\left({ }^{4} S_{3 / 2}^{o} \rightarrow{ }^{4} P_{1 / 2}\right)_{\mathrm{O} \text { II }} \\
& =29650 \mathrm{~cm}^{-1}, \\
A_{I I I} & =0.59 \omega\left(3 s_{1 / 2} \rightarrow 3 p_{1 / 2}\right)_{\mathrm{Al} \mathrm{II}}-0.41 \omega\left(3 s_{1 / 2} \rightarrow 3 p_{3 / 2}\right)_{\mathrm{Al} \mathrm{II}} \\
& =6781 \mathrm{~cm}^{-1},
\end{aligned}
$$

$$
\begin{aligned}
A_{I V} & =0.68 \omega\left(3 s_{1 / 2} \rightarrow 3 p_{1 / 2}\right)_{\mathrm{Al} \mathrm{III}}-0.32 \omega\left(3 s_{1 / 2} \rightarrow 3 p_{3 / 2}\right)_{\mathrm{Al} \mathrm{III}} \\
& =19465 \mathrm{~cm}^{-1} .
\end{aligned}
$$

For ${ }^{55} \mathrm{Mn}$ we can form several anchors, such as

$$
\begin{aligned}
A_{V} & =0.67 \omega\left({ }^{7} S_{3} \rightarrow{ }^{7} P_{4}^{o}\left[3 d^{5} 4 p\right]\right)_{\mathrm{Mn} \mathrm{II}}+0.33 \omega\left({ }^{7} S_{3}\right. \\
& \left.\rightarrow{ }^{7} P_{4}^{o}\left[3 d^{4} 4 s 4 p\right]\right)_{\mathrm{Mn} \mathrm{II}}=53699 \mathrm{~cm}^{-1} .
\end{aligned}
$$

The numerical factors in Eqs. (8)-(11) are formed from the $q$ factors for two transitions and are normalized to unity. In the leading order $A_{I I}-A_{V}$ do not depend on $\alpha$ and can be used as anchors.

Other elements in Table I have more than one isotope. Strong correlation between the factors $q$ and $k_{\mathrm{MS}}$ for $\mathrm{Fe}$ II allows us to form several combinations of frequencies suitable as anchors. It could be possible to make anchors from practically any pair of lines in $\mathrm{Fe}_{\text {II }}$ which include the line $62172 \mathrm{~cm}^{-1}$, for example, 
TABLE I. Results of the calculations of the $\alpha$-variation parameters $q$ [19-22] and the isotope shift parameters $k_{\mathrm{MS}}$ [10,12] for the transitions used in astrophysical surveys. The error bars are given in parentheses where available. Column $\Delta \omega_{\alpha}$ gives the shifts that correspond to the $\alpha$ variation observed in [4]. Column $\Delta \omega_{1}$ shows the line shift caused by a $10 \%$ abundance transfer from the leading isotope to the next to leading (by abundance). Similarly, column $\Delta \omega_{2}$ presents the line shift for the case when natural abundance is substituted by a single leading isotope.

\begin{tabular}{|c|c|c|c|c|c|c|c|c|}
\hline \multirow{2}{*}{$\frac{\text { Ion }}{\mathrm{O}_{\text {II }}}$} & Transition & \multirow{2}{*}{$\begin{array}{c}\begin{array}{c}\omega_{0} \\
\left(\mathrm{~cm}^{-1}\right)\end{array} \\
119873\end{array}$} & \multirow{2}{*}{$\frac{\begin{array}{c}q \\
\left(\mathrm{~cm}^{-1}\right)\end{array}}{346}$} & \multirow[t]{2}{*}{$\begin{array}{l}k_{\mathrm{MS}} \\
\left(\mathrm{cm}^{-1} \mathrm{amu}\right)\end{array}$} & \multicolumn{3}{|c|}{$\begin{array}{c}\Delta \omega_{\alpha} \Delta \omega_{1} \Delta \omega_{2} \\
\left(10^{-3} \mathrm{~cm}^{-1}\right)\end{array}$} & \multirow{2}{*}{$\frac{\text { References }}{[21]}$} \\
\hline & ${ }^{4} S_{3 / 2}^{o}\left[2 s^{2} 2 p^{4}\right] \rightarrow{ }^{4} P_{5 / 2}\left[2 s 2 p^{4}\right]$ & & & & -4 & & 0 & \\
\hline & $\rightarrow{ }^{4} P_{3 / 2}\left[2 s 2 p^{4}\right]$ & 120000 & 489 & & -6 & & 0 & [21] \\
\hline & $\rightarrow{ }^{4} P_{1 / 2}\left[2 s 2 p^{4}\right]$ & 120083 & 574 & & -7 & & 0 & {$[21]$} \\
\hline $\mathrm{Mg}_{\mathrm{I}}$ & ${ }^{1} S_{0}\left[3 s^{2}\right] \rightarrow{ }^{1} P_{1}^{o}[3 s 3 p]^{\mathrm{a}}$ & 35051 & 86 & $14.5(0.3)$ & -1 & 5 & -8 & {$[19,23]$} \\
\hline \multirow[t]{2}{*}{$\mathrm{Mg}_{\text {II }}$} & $3 s_{1 / 2} \rightarrow 3 p_{1 / 2}$ & 35669 & 120 & $32.1(0.4)$ & -1 & 10 & -16 & {$[10,19]$} \\
\hline & $\rightarrow 3 p_{3 / 2}$ & 35761 & 211 & $32.1(0.2)$ & -2 & 10 & -16 & {$[10,19]$} \\
\hline \multirow[t]{2}{*}{$\mathrm{Al}$ II } & ${ }^{1} S_{0}\left[3 s^{2}\right] \rightarrow{ }^{3} P_{0}[3 s 3 p]$ & 37393 & $146(15)$ & & -2 & & 0 & {$[22]$} \\
\hline & $\rightarrow{ }^{3} P_{1}[3 s 3 p]$ & 37454 & $211(20)$ & & -2 & & 0 & {$[22]$} \\
\hline \multirow[t]{2}{*}{$\mathrm{Al}$ III } & $3 s_{1 / 2} \rightarrow 3 p_{1 / 2}$ & 53682 & 216 & & -2 & & 0 & [19] \\
\hline & $\rightarrow 3 p_{3 / 2}$ & 53920 & 464 & & -5 & & 0 & [19] \\
\hline \multirow[t]{2}{*}{ Si II } & ${ }^{2} P_{1 / 2}^{o}\left[3 s^{2} 3 p\right] \rightarrow{ }^{2} D_{3 / 2}\left[3 s 3 p^{2}\right]$ & 55309 & $520(30)$ & $63(15)$ & -6 & 8 & -9 & {$[10,20]$} \\
\hline & $\rightarrow{ }^{2} S_{1 / 2}\left[2 s^{2} 4 s\right]$ & 65495 & $50(30)$ & $-13(20)$ & -1 & -2 & 2 & {$[10,20]$} \\
\hline \multirow[t]{2}{*}{ Si IV } & $3 s_{1 / 2} \rightarrow 3 p_{1 / 2}$ & 71290 & 346 & $90.3(0.4)$ & -4 & 11 & -13 & {$[10,19]$} \\
\hline & $\rightarrow 3 p_{3 / 2}$ & 71750 & 862 & $89.6(0.2)$ & -10 & 11 & -13 & {$[10,19]$} \\
\hline \multirow[t]{2}{*}{ Ti II } & ${ }^{4} F_{3 / 2}\left[3 d^{2} 4 s\right] \rightarrow{ }^{4} F_{3 / 2}^{o}\left[3 d^{2} 4 p\right]$ & 30837 & $541(50)$ & $20(4)$ & -6 & -2 & 1 & {$[12,21]$} \\
\hline & $\rightarrow{ }^{4} F_{5 / 2}^{o}\left[3 d^{2} 4 p\right]$ & 30959 & $673(70)$ & $20(4)$ & -8 & -2 & 1 & {$[12,21]$} \\
\hline \multirow[t]{3}{*}{$\mathrm{Cr}_{\text {II }}$} & ${ }^{6} S_{5 / 2}\left[3 d^{5}\right] \rightarrow{ }^{6} P_{3 / 2}^{o}\left[3 d^{4} 4 p\right]$ & 48399 & $-1360(150)$ & $-63(40)$ & 16 & -2 & 1 & {$[12,20]$} \\
\hline & $\rightarrow{ }^{6} P_{5 / 2}^{o}\left[3 d^{4} 4 p\right]$ & 48491 & $-1280(150)$ & $-63(40)$ & 15 & -2 & 1 & {$[12,20]$} \\
\hline & $\rightarrow{ }^{6} P_{7 / 2}^{o}\left[3 d^{4} 4 p\right]$ & 48632 & $-1110(150)$ & $-63(40)$ & 13 & -2 & 1 & {$[12,20]$} \\
\hline \multirow[t]{6}{*}{ Mn II } & ${ }^{7} S_{3}\left[3 d^{5} 4 s\right] \rightarrow{ }^{7} P_{2}^{o}\left[3 d^{5} 4 p\right]$ & 38366 & $869(90)$ & & -10 & & 0 & {$[21]$} \\
\hline & $\rightarrow{ }^{7} P_{3}^{o}\left[3 d^{5} 4 p\right]$ & 38543 & $1030(100)$ & & -12 & & 0 & {$[21]$} \\
\hline & $\rightarrow{ }^{7} P_{4}^{o}\left[3 d^{5} 4 p\right]$ & 38807 & $1276(100)$ & & -15 & & 0 & {$[21]$} \\
\hline & $\rightarrow{ }^{7} P_{2}^{o}\left[3 d^{4} 4 s 4 p\right]$ & 83255 & $-3033(300)$ & & 35 & & 0 & {$[21]$} \\
\hline & $\rightarrow{ }^{7} P_{3}^{o}\left[3 d^{4} 4 s 4 p\right]$ & 83376 & $-2825(300)$ & & 32 & & 0 & {$[21]$} \\
\hline & $\rightarrow{ }^{7} P_{4}^{o}\left[3 d^{4} 4 s 4 p\right]$ & 83529 & $-2556(300)$ & & 29 & & 0 & {$[21]$} \\
\hline \multirow[t]{7}{*}{$\mathrm{Fe}_{\text {II }}$} & ${ }^{6} D_{9 / 2}\left[3 d^{6} 4 s\right] \rightarrow{ }^{6} D_{9 / 2}^{o}\left[3 d^{6} 4 p\right]^{\mathrm{b}}$ & 38459 & $1330(150)$ & $60(20)$ & -15 & -4 & 2 & {$[12,20]$} \\
\hline & $\rightarrow{ }^{6} D_{7 / 2}^{o}\left[3 d^{6} 4 p\right]^{\mathrm{b}}$ & 38660 & $1490(150)$ & $60(20)$ & -17 & -4 & 2 & {$[12,20]$} \\
\hline & $\rightarrow{ }^{6} F_{11 / 2}^{o}\left[3 d^{6} 4 p\right]^{\mathrm{b}}$ & 41968 & $1460(150)$ & $63(20)$ & -17 & -4 & 2 & {$[12,20]$} \\
\hline & $\rightarrow{ }^{6} F_{9 / 2}^{o}\left[3 d^{6} 4 p\right]^{\mathrm{b}}$ & 42115 & $1590(150)$ & $63(20)$ & -18 & -4 & 2 & {$[12,20]$} \\
\hline & $\rightarrow{ }^{6} P_{7 / 2}^{o}\left[3 d^{6} 4 p\right]^{\mathrm{b}}$ & 42658 & $1210(150)$ & $60(20)$ & -14 & -4 & 2 & {$[12,20]$} \\
\hline & $\rightarrow{ }^{4} F_{7 / 2}^{o}\left[3 d^{6} 4 p\right]$ & 62066 & $1100(300)$ & $67(40)$ & -13 & -4 & 2 & {$[12,20]$} \\
\hline & $\rightarrow{ }^{6} P_{7 / 2}^{o}\left[3 d^{5} 4 s 4 p\right]^{\mathrm{b}}$ & 62172 & $-1300(300)$ & $-67(40)$ & 15 & 4 & -2 & {$[12,20]$} \\
\hline \multirow[t]{3}{*}{$\mathrm{Ni}$ II } & ${ }^{2} D_{5 / 2}\left[3 d^{9}\right] \rightarrow{ }^{2} F_{7 / 2}^{o}\left[3 d^{8} 4 p\right]$ & 57080 & $-700(250)$ & $-77(50)$ & 8 & -4 & 18 & {$[12,20]$} \\
\hline & $\rightarrow{ }^{2} D_{5 / 2}^{o}\left[3 d^{8} 4 p\right]$ & 57420 & $-1400(250)$ & $-77(50)$ & 16 & -4 & 18 & {$[12,20]$} \\
\hline & $\rightarrow{ }^{2} F_{5 / 2}^{o}\left[3 d^{8} 4 p\right]$ & 58493 & $-20(250)$ & $-77(50)$ & 0 & -4 & 18 & {$[12,20]$} \\
\hline \multirow[t]{2}{*}{$\mathrm{Zn}$ II } & $4 s_{1 / 2} \rightarrow 4 p_{1 / 2}$ & 48481 & $1584(25)$ & $70.3(2.3)$ & -18 & 3 & -24 & {$[10,20]$} \\
\hline & $\rightarrow 4 p_{3 / 2}$ & 49355 & $2490(25)$ & $69.3(2.3)$ & -28 & 3 & -24 & {$[10,20]$} \\
\hline Ge II & $4 p_{1 / 2} \rightarrow 5 s_{1 / 2}$ & 62403 & 607 & $-0.7(2.3)$ & -7 & 0 & 0 & {$[10,19]$} \\
\hline
\end{tabular}

${ }^{\mathrm{a}}$ For this transition we give the experimental value for $k_{\mathrm{MS}}$ from [23].

${ }^{\mathrm{b}}$ The lines of Fe II used by Quast et al. [5]. 


$$
\begin{aligned}
A_{V I} & =0.5 \omega\left({ }^{6} D_{9 / 2} \rightarrow{ }^{6} D_{9 / 2}^{o}\right)_{\mathrm{Fe} \mathrm{II}}+0.5 \omega\left({ }^{6} D_{9 / 2} \rightarrow{ }^{6} P_{7 / 2}^{o}\right)_{\mathrm{Fe} \mathrm{II}} \\
& =50316 \mathrm{~cm}^{-1} .
\end{aligned}
$$

Now, when we have several anchors to exclude the cosmological redshift, we can look for the combinations of frequencies that are sensitive to only the mass shift or to the variation of $\alpha$. These combinations can be used as "probes" of isotope abundances and $\alpha$ variation. For example, the combination

$$
\begin{aligned}
P_{I} & =0.64 \omega\left(3 s_{1 / 2} \rightarrow 3 p_{1 / 2}\right)_{\mathrm{Mg} \mathrm{II}}-0.36 \omega\left(3 s_{1 / 2} \rightarrow 3 p_{3 / 2}\right)_{\mathrm{Mg} \mathrm{II}} \\
& =9740 \mathrm{~cm}^{-1}
\end{aligned}
$$

is insensitive to $\alpha$ variation and can serve as a probe of the abundances of $\mathrm{Mg}$ isotopes in distant absorbers.

Note that even though $q$ factors for $\mathrm{Mg}$ are relatively small, it is still necessary to use $P_{I}$ as a probe, rather than individual lines. Since $\alpha$ variation on the scale of $\Delta \alpha / \alpha \approx$ $-0.6 \times 10^{-5}$ is currently not excluded, we can expect the shift $\delta \omega\left(3 s_{1 / 2} \rightarrow 3 p_{3 / 2}\right) \approx-0.002 \mathrm{~cm}^{-1}$, which is comparable to the current sensitivity of observations to the frequency shifts.

By analogy with $\mathrm{Mg}$ II it is possible to form combinations for the fine-structure doublets in $\mathrm{Si}$ IV and $\mathrm{Zn}$ II:

$$
\begin{aligned}
P_{I I} & =0.71 \omega\left(3 s_{1 / 2} \rightarrow 3 p_{1 / 2}\right)_{\mathrm{Si} \mathrm{IV}}-0.29 \omega\left(3 s_{1 / 2} \rightarrow 3 p_{3 / 2}\right)_{\mathrm{Si} \mathrm{IV}} \\
& =30381 \mathrm{~cm}^{-1}, \\
P_{I I I} & =0.61 \omega\left(4 s_{1 / 2} \rightarrow 4 p_{1 / 2}\right)_{\mathrm{Zn} \mathrm{II}}-0.39 \omega\left(4 s_{1 / 2} \rightarrow 4 p_{3 / 2}\right)_{\mathrm{Zn} \mathrm{II}} \\
& =10423 \mathrm{~cm}^{-1} .
\end{aligned}
$$

For most other transitions in Table I there is strong correlation between parameters $q$ and $k_{\mathrm{MS}}$, and we cannot exclude the dependence on $\alpha$. The only exception is $\mathrm{Ni}$ II, where the transition with frequency $58493 \mathrm{~cm}^{-1}$ is insensitive to $\alpha$ variation and two other transitions have different parameters $q$. That gives us two more probes of isotope abundances:

$$
\begin{gathered}
P_{I V}=\omega\left({ }^{2} D_{5 / 2} \rightarrow{ }^{2} F_{5 / 2}^{o}\right)_{\mathrm{Ni} \mathrm{II}}=58493 \mathrm{~cm}^{-1}, \\
P_{V}=0.67 \omega\left({ }^{2} D_{5 / 2} \rightarrow{ }^{2} F_{7 / 2}^{o}\right)_{\mathrm{Ni} \mathrm{II}}-0.33 \omega\left({ }^{2} D_{5 / 2} \rightarrow{ }^{2} D_{5 / 2}^{o}\right)_{\mathrm{Ni} \mathrm{III}} \\
=18952 \mathrm{~cm}^{-1} .
\end{gathered}
$$

The same method can be applied for building combinations that are insensitive to isotope shift and can serve as probes for the variation of $\alpha$. Above we suggested that several combinations of spectral lines of $\mathrm{O}, \mathrm{Al}$, and $\mathrm{Mn}$ ions can be used as anchors. These lines themselves can also be introduced as $\alpha$-dependent probes. Indeed, these elements have only one stable isotope each and therefore have no isotope shifts. Here, manganese is the best probe, as it has the big-

\begin{tabular}{|c|c|c|c|}
\hline Ion & $\begin{array}{c}\omega \\
\mathrm{cm}^{-1}\end{array}$ & $\begin{array}{r}q \\
\mathrm{~cm}^{-1}\end{array}$ & $\begin{array}{l}\text { Frequency } \\
\text { combinations }\end{array}$ \\
\hline \multirow[t]{3}{*}{ O II } & 119873 & 346 & $\omega\left({ }^{4} S_{3 / 2}^{o} \rightarrow{ }^{4} P_{5 / 2}\right)$ \\
\hline & 120000 & 489 & $\omega\left({ }^{4} S_{3 / 2}^{o} \rightarrow{ }^{4} P_{3 / 2}\right)$ \\
\hline & 120083 & 574 & $\omega\left({ }^{4} S_{3 / 2}^{o} \rightarrow{ }^{4} P_{1 / 2}\right)$ \\
\hline \multirow[t]{2}{*}{$\mathrm{Al}$ II } & 37393 & 146 & $\omega\left({ }^{1} S_{0} \rightarrow{ }^{3} P_{0}\right)$ \\
\hline & 36454 & 211 & $\omega\left({ }^{1} S_{0} \rightarrow{ }^{3} P_{1}\right)$ \\
\hline \multirow[t]{2}{*}{$\mathrm{Al}$ III } & 53682 & 216 & $\omega\left(s_{1 / 2} \rightarrow 3 p_{1 / 2}\right)$ \\
\hline & 53920 & 464 & $\omega\left(s_{1 / 2} \rightarrow 3 p_{3 / 2}\right)$ \\
\hline \multirow[t]{2}{*}{ Si IV } & 230 & 258 & $(1 / 2) \omega\left(3 s_{1 / 2} \rightarrow 3 p_{3 / 2}\right)$ \\
\hline & 38366 & 869 & $\begin{array}{r}-(1 / 2) \omega\left(3 s_{1 / 2} \rightarrow 3 p_{1 / 2}\right) \\
\omega\left({ }^{7} S_{3} \rightarrow{ }^{7} P_{2}\left[3 d^{5} 4 p\right]\right)\end{array}$ \\
\hline \multirow{5}{*}{ Mn II } & 38543 & 1030 & $\omega\left({ }^{7} S_{3} \rightarrow{ }^{7} P_{3}\left[3 d^{5} 4 p\right]\right)$ \\
\hline & 38807 & 1276 & $\omega\left({ }^{7} S_{3} \rightarrow{ }^{7} P_{4}\left[3 d^{5} 4 p\right]\right)$ \\
\hline & 83255 & -3033 & $\omega\left({ }^{7} S_{3} \rightarrow{ }^{7} P_{2}\left[3 d^{4} 4 s 4 p\right]\right)$ \\
\hline & 83376 & -2825 & $\omega\left({ }^{7} S_{3} \rightarrow{ }^{7} P_{3}\left[3 d^{4} 4 s 4 p\right]\right)$ \\
\hline & 83529 & -2556 & $\omega\left({ }^{7} S_{3} \rightarrow{ }^{7} P_{4}\left[3 d^{4} 4 s 4 p\right]\right)$ \\
\hline \multirow[t]{2}{*}{ Ni II } & 170 & -350 & $(1 / 2) \omega\left({ }^{2} D_{5 / 2} \rightarrow{ }^{6} D_{5 / 2}^{o}\right)$ \\
\hline & 537 & 690 & $\begin{aligned}-(1 / 2) \omega\left({ }^{2} D_{5 / 2}\right. & \left.\rightarrow{ }^{6} F_{7 / 2}^{o}\right) \\
(1 / 2) \omega\left({ }^{2} D_{5 / 2}\right. & \left.\rightarrow{ }^{6} F_{5 / 2}^{o}\right) \\
-(1 / 2) \omega\left({ }^{2} D_{5 / 2}\right. & \left.\rightarrow{ }^{6} D_{5 / 2}^{o}\right)\end{aligned}$ \\
\hline \multirow[t]{2}{*}{$\mathrm{Zn}$ II } & 437 & 453 & $(1 / 2) \omega\left(4 s_{1 / 2} \rightarrow 4 p_{3 / 2}\right)$ \\
\hline & & & $-(1 / 2) \omega\left(4 s_{1 / 2} \rightarrow 4 p_{1 / 2}\right)$ \\
\hline $\mathrm{Ge}_{\text {II }}$ & 62403 & 607 & $\omega\left(4 p_{1 / 2} \rightarrow 5 s_{1 / 2}\right)$ \\
\hline
\end{tabular}
gest absolute value of $q$. Ge II is also a good probe because of a very low value of $k_{\mathrm{MS}}$ of the only transition $4 p_{1 / 2} \rightarrow 5 s_{1 / 2}$, listed in Table I.

Other ions from Table I are sensitive to isotope shifts. Still it is possible to make several combinations to exclude this dependence. $\mathrm{Mg}$ II, Si II, and $\mathrm{Ti}$ II have relatively low values of $q$ and would not make good probes. For remaining ions $k_{\mathrm{MS}}$ is practically the same for all transitions of interest. Hence, we can form a probe by taking the difference of any
TABLE II. $\alpha$-variation probes, insensitive to isotope shifts.

two frequencies. The results are given in Table II, where we introduced normalization factors as above.

For $\mathrm{Fe}$ II and $\mathrm{Cr}$ II it is impossible to exclude isotope dependence because of the correlations between $q$ and $k_{\mathrm{MS}}$. In both cases isotopic effects are relatively small. That allows one to use these ions to test $\alpha$ variation on the level reported in [4], but not on the level of the result [6].

\section{DISCUSSION OF SENSITIVITY}

Let us use Eq. (2) and Table I to estimate typical sensitivity to the frequency shifts in modern astrophysical studies. The paper [5] is based only on the analysis of the lines of Fe II. From Table I we see that for all transitions in this ion $|q| \approx 1300 \mathrm{~cm}^{-1}$. Equation (1b) then gives statistical accuracy $\Delta \omega \approx 0.005 \mathrm{~cm}^{-1}$. Two other groups [4,6] use more ions and obtain higher statistical accuracy. This accuracy is achieved by averaging results for many ions, so we will still use the same estimate of the achievable accuracy for the frequency shifts in astrophysical observations for a particular ion:

$$
\Delta \omega \approx 0.005 \mathrm{~cm}^{-1} .
$$

Now we estimate frequency shifts for the probes $P_{I}-P_{V}$ and compare them with current sensitivity (17). To do this we need to specify the way in which isotope abundances are 
changed. We assume that the intensity of the line of the leading isotope is transferred to the next to leading one. If there are two comparable weaker lines, we assume that they are equally increased. Within this simple model we calculate the shifts of the centers of gravity of the lines and the resulting shift of the probe. Comparison of this shift to the modern frequency sensitivity (17) gives us the sensitivity to the isotope abundances.

$\mathrm{Mg}$ has three stable isotopes $A=24,25$, and 26 with modern abundances 79:10:11. Suppose that in the early universe it was $(79-x):(10+x / 2):(11+x / 2)$. Using the factors $k_{\mathrm{MS}}$ from Table I and ignoring the volume shift and saturation effects, we can estimate the corresponding shift of the center of gravity of the probe (12):

$$
\delta P_{I}=-0.27 \frac{3 k_{\mathrm{MS}} x}{200 A^{2}} \approx 0.00023 x \mathrm{~cm}^{-1} .
$$

For $\mathrm{Si}$ and $\mathrm{Zn}$ there are also two comparable admixtures to the leading isotope. If we assume $(92-x):(5+x / 2):(3$ $+x / 2)$ abundances for $\mathrm{Si}$ and $(49-y): 0:(28+y / 2): 4:(19$ $+y / 2): 0: 1$ abundances for $\mathrm{Zn}$, we get

$$
\begin{gathered}
\delta P_{I I}=-0.43 \frac{3 k_{\mathrm{MS}} x}{200 \times 28^{2}} \approx 0.00074 x \mathrm{~cm}^{-1}, \\
\delta P_{I I I}=-0.22 \frac{3 k_{\mathrm{MS}} y}{100 \times 64^{2}} \approx 0.00011 y \mathrm{~cm}^{-1} .
\end{gathered}
$$

$\mathrm{Ni}$ has only one dominant admixture to the leading isotope, so we assume $(68-x): 0:(26+x): 1: 4: 0: 1$ abundances for Ni. That gives

$$
\delta P_{I V}=-\frac{2 k_{\mathrm{MS}} x}{100 \times 58^{2}} \approx 0.00046 x \mathrm{~cm}^{-1},
$$

while the probe $P_{V}$ is three times less sensitive.
Comparing Eqs. (18)-(21) with Eq. (17) we see that current sensitivity allows detection of a $22 \%$ change in the abundance of the isotope ${ }^{24} \mathrm{Mg}$, a $7 \%$ change for the isotope ${ }^{28} \mathrm{Si}, 45 \%$ for ${ }^{64} \mathrm{Zn}$, and an $11 \%$ change in the abundance of ${ }^{58} \mathrm{Ni}$.

\section{CONCLUSIONS}

In this paper we analyze the sensitivity of modern astrophysical surveys that search for $\alpha$ variation to isotopic effects and come to the following conclusions.

Isotopic frequency shifts are generally likely to be of the same order of magnitude as the present experimental sensitivity. Therefore, isotope shifts are an important source of systematic errors.

It is possible to eliminate isotopic effects by taking proper combinations of transition frequencies. These combinations depend on the calculated mass shift coefficients. At present not all of them are known with a sufficient accuracy. The corresponding theory can be improved by applying the method suggested in $[15,16]$. New experimental data on the isotope shifts are also highly desirable.

As a by-product of the search for $\alpha$ variation it is possible to get information about isotopic evolution of several elements.

The accuracy of the surveys that use only lines of $\mathrm{Fe}_{\text {II }}$ is limited by the isotopic effects on the level $\delta \alpha / \alpha \sim 10^{-6}$. This estimate is based on rather conservative assumptions about possible isotope evolution.

\section{ACKNOWLEDGMENTS}

We thank S. A. Levshakov for very helpful comments on the manuscript. M.K. is grateful to M. Murphy and D. A. Varshalovich for valuable discussions. This work is supported by the Russian Foundation for Basic Research, Grant No. 02-0216387, and the Australian Research Council.
[1] V. A. Dzuba, V. V. Flambaum, and J. K. Webb, Phys. Rev. Lett. 82, 888 (1999).

[2] J. K. Webb, V. V. Flambaum, C. W. Churchill, M. J. Drinkwater, and J. D. Barrow, Phys. Rev. Lett. 82, 884 (1999).

[3] J. K. Webb, M. T. Murphy, V. V. Flambaum, V. A. Dzuba, J. D. Barrow, C. W. Churchill, J. X. Prochaska, and A. M. Wolfe, Phys. Rev. Lett. 87, 091301 (2001).

[4] M. T. Murphy, V. V. Flambaum, J. K. Webb, V. A. Dzuba, J. X. Prochaska, and A. M. Wolfe, Mon. Not. R. Astron. Soc. 345, 609 (2003).

[5] R. Quast, D. Reimers, and S. A. Levshakov, Astron. Astrophys. 414, L7 (2004).

[6] R. Srianand, H. Chand, P. Petitjean, and B. Aracil, Phys. Rev. Lett. 92, 121302 (2004).

[7] S. A. Levshakov, Mon. Not. R. Astron. Soc. 269, 339 (1994).

[8] D. A. Varshalovich, A. Y. Potekhin, and A. V. Ivanchik, Phys. Scr., T 95, 76 (2001).
[9] T. P. Ashenfelter, G. J. Mathews, and K. A. Olive, e-print astro-ph/0404257.

[10] J. C. Berengut, V. A. Dzuba, and V. V. Flambaum, Phys. Rev. A 68, 022502 (2003).

[11] J. C. Berengut, V. A. Dzuba, V. V. Flambaum, and M. G. Kozlov, Phys. Rev. A 69, 044102 (2004).

[12] J. C. Berengut, V. A. Dzuba, V. V. Flambaum, and M. G. Kozlov (unpublished).

[13] I. I. Sobelman, Atomic Spectra and Radiative Transitions (Springer-Verlag, Berlin, 1979).

[14] W. H. King, Isotope Shifts in Atomic Spectra (Plenum Press, New York, 1984).

[15] V. A. Dzuba, V. V. Flambaum, and M. G. Kozlov, JETP Lett. 63, 882 (1996).

[16] V. A. Dzuba, V. V. Flambaum, and M. G. Kozlov, Phys. Rev. A 54, 3948 (1996).

[17] I. I. Tupitsyn et al., Phys. Rev. A 68, 022511 (2003).

[18] M. S. Safronova and W. R. Johnson, Phys. Rev. A 64, 052501 
(2001)

[19] V. A. Dzuba, V. V. Flambaum, and J. K. Webb, Phys. Rev. A 59, 230 (1999).

[20] V. A. Dzuba, V. V. Flambaum, M. G. Kozlov, and M. Marchenko, Phys. Rev. A 66, 022501 (2002).
[21] J. C. Berengut, V. A. Dzuba, V. V. Flambaum, and M. V. Marchenko, e-print physics/0404008.

[22] E. J. Angstmann, V. A. Dzuba, and V. V. Flambaum, e-print physics/0404042.

[23] L. Hallstadius, Z. Phys. A 291, 203 (1979). 